www.jmscr.igmpublication.org

Impact Factor (SJIF): 6.379

Index Copernicus Value: 79.54

ISSN (e)-2347-176x ISSN (p) 2455-0450

crossrefDOI: https://dx.doi.org/10.18535/jmscr/v6i10.181

Journal Of Medical Science And Clinical Research

\title{
Neutrophil Lymphocyte Ratio (NLR) in Patients with Lung Cancer - An Index of Cancer Related Systemic Inflammation
}

Authors

Raseela Karunakaran ${ }^{1}$, Santhosh Surendran ${ }^{2}$, Mithun Harold Thomas ${ }^{3 *}$

${ }^{1}$ Department of Respiratory Medicine, Government TD Medical College, Alappuzha, Kerala, India

${ }^{2}$ Department of Anaesthesiology, Government TD Medical College, Alappuzha, Kerala, India

${ }^{3}$ Department of Gastroenterology, Government Medical College, Kozhikode, Kerala, India

*Corresponding Author

Mithun Harold Thomas

Senior Resident, Department of Gastroenterology, Government Medical College, Kozhikode, Kerala, India Phone number: 9809587773, Email: drmithunharold@gmail.com

\begin{abstract}
Introduction: Neutrophilia has been associated with many chronic inflammatory lung diseases, lung cancer and systemic inflammatory diseases. A common denominator of inflammation in these entities is Th 17 type of $C D 4+T$ cell adaptive immunity. Neutrophil infiltration of tumour microenvironment and peripheral blood neutrophilia has become a focus of attraction in recent research on chronic inflammatory diseases. Many studies have shown that increased neutrophil:lymphocyte ratio(NLR) and advanced lung cancer inflammation index (ALI) are poor prognostic indicators in cancers.

Aims: This study aims to find out the neutrophil: lymphocyte ratio in lung cancer patients.

Materials and Methods: This study is a retrospective analysis conducted in the Department of Pulmonary medicine in a tertiary care centre in south Kerala. The case records of 50 patients, histologically diagnosed as lung cancer were analysed. NLR was calculated from routine blood examination results.

Results: The total number of patients included in the study was 50.The most common histological type of lung cancer was adenocarcinoma in both males and females of all ages. Neutrophil lymphocyte ratio was high in all patients, irrespective of the histological type. Mean value of NLR was 5.33 (reference range, 2.15 or less) ${ }^{(1)}$

Conclusion: This study shows that neutrophils are the predominant leukocytes in peripheral blood of lung cancer patients, supporting the concept of ongoing injury and repair processes in the pathogenesis of inflammation in lung cancer. NLR is a simple and inexpensive bed side biomarker of systemic inflammation in lung cancer patients.

Keywords: Lung cancer, neutrophil, lymphocyte, NLR.
\end{abstract}

\section{Introduction}

Lung cancer is a major cause of cancer related mortality worldwide, comprising $17 \%$ of the total new cancer cases and $23 \%$ of the total cancer deaths. It forms the leading cause of cancer death among males, in both developed and developing countries. Among females, lung cancer has surpassed breast cancer as the leading cause of cancer death in more developed countries. Now it is well accepted that many lung diseases such as 
cancer, COPD, asthma and IPF are inflammatory lung diseases that share the common pathway of inflammation and aberrant repair ${ }^{(2)}$ Inflammation is the natural response of body to any sort of injury(pathogen, allergen, toxins, products from tissue damage), and is mediated by the innate and adaptive immune systems. Innate immunity together with adaptive immunity makes up the natural host defensive barrier; composed of epithelial endothelial barriers, germline encoded receptors, immune organs, immune inflammatory cells, and cytokines. Multicellular organisms possess germline encoded surveillance systems to detect these signals. Innate immunity aroused approximately 600 million years ago and is evolutionarily conserved across different forms of life species. Adaptive immunity came later during evolution and is present only in vertebrates ${ }^{(3)}$.The host immune response in inflammation is a well co-ordinated signalling programme initiated by the innate immune system , complemented by the adaptive immune system and carried out via a complex neurohumoral- immune axis, executed at the cellular level through the cytokine signalling directed network system.

Neutrophils, the first line phagocytic cells attracted to the site of inflammation form the major cellular players in acute inflammation. But these short lived cells are known to have afore most role in the inflammatory process in various chronic pulmonary diseases such as cancer ${ }^{(4)}$, COPD, asthma, cystic fibrosis and $\operatorname{IPF}^{(5,6)}$. Neutrophils in the tumor micro environment termed tumor associated neutrophils (TANs), have complex interaction with macrophages in the tumor milieu and have an unquestionable rolein tumor progression, angiogenesis and metastasis in various types of cancers in humans. The exact role of neutrophils in cancer related inflammation is not yet unveiled.

Haematological indices such as Neutrophil to lymphocyte ratio (NLR), platelet to lymphocyte ratio (PLR), lymphocyte to monocyte ratio (LMR), advanced lung cancer inflammation index ${ }^{(7)}$ and serum levels of acute phase protein and C-reactive proteins (CRP) have been found to be useful markers of inflammation in lung cancer ${ }^{(8-11)}$.

\section{Materials and Methods}

This study was a retrospective analysis conducted in the Department of Pulmonary medicine in a tertiary care centre. The case records of 50 patients with histological diagnosis of lung cancer were analysed. Total leukocyte count, neutrophil and lymphocyte counts were noted. NLR ratio was calculated from the data collected.

Exclusion criteria: Patients with acute infections, likely to have peripheral blood neutrophilia were excluded from the study.

\section{Results}

The total number of patients included in the study was 50, out of which 38 patients were male and 12 patients were females. The number of adenocarcinoma was 34 and squamous cell carcinoma was 8 , while 8 cases were not differentiated regarding the cell type. In all the groups of cancer patients the NLR was high irrespective of the histological type(mean value 5.33). On case of carcinoid tumor was diagnosed in a female patient which also showed high NLR. The most common comorbidities were COPD, diabetes and tuberculosis. Among the comorbidities, COPD and active Tuberculosis, which are definite risk factors for lung cancer, show high NLR compared to others, 4.79 and 9.72 respectively, reflecting the higher inflammatory status in these conditions that may be contributing to cancer related inflammation.

Table 1: Results

\begin{tabular}{|l|c|c|}
\hline Total Number of patients & 50 & NLR \\
\hline Male & 38 & 5.76 \\
\hline Female & 12 & 3.41 \\
\hline Histological type & \multicolumn{2}{|l|}{} \\
\hline Adenocarcinoma & 34 & 5.42 \\
\hline Squamous cell carcinoma & 8 & 3.58 \\
\hline Undifferentiated & 8 & 6.65 \\
\hline Carcinoid & 1 & 2.43 \\
\hline Comorbidity & 9 & 4.79 \\
\hline COPD & 5 & 3.63 \\
\hline Diabetes mellitus & 2 & 3.09 \\
\hline Healed PulmonaryTB & 2 & 9.72 \\
\hline Active tuberculosis &
\end{tabular}

\section{Discussion}

Blood neutrophils are relatively short-lived cells and are the most abundant leucocyte population in 
human blood (40-60\%) constituting an important first line defense against infections. Functionally intact neutrophils are essential for life in mammals, including humans. Neutrophils released into the circulation from the bone marrow is constantly generated by granulopoesis. Nearly $10^{11}$ cells are generated and released into the circulation every day. Complete blood count (CBC) with differential WBC count is one of the most common and simple laboratory tests performed in the clinic. The absolute count of neutrophils, lymphocytes, and monocytes reflects the inflammatory response and overall immune status of the body. Peripheral blood prognostic inflammatory markers including the neutrophil-to-lymphocyte

ratio (NLR), lymphocyte-to-monocyte ratio (LMR), platelet-to-lymphocyte ratio (PLR) are simple and inexpensive markers of inflammatory status in cancer patients, and has been found to have correlation with treatment response and prognosis in various types of cancers ${ }^{(8,10,11)}$. However, there are a limited number of reports about the relationship between NLR and lung cancer among our patients.

\section{Neutrophils in resolution of inflammation}

The histologic hallmark of resolution of inflammation is disappearance of neutrophils from the tissue ${ }^{(12)}$. Persistence of neutrophils in tissues accelerate the inflammatory process by tissue damage via reactive oxygen species (ROS) and extracellular release of enzymes contained in neutrophil granules and cytokine release. Neutrophils are short life span cells but they can live for weeks in cancer tissues and promote tumor progression $^{(13)}$. Apoptotic neutrophils are taken up by macrophages and carried to lymph nodes, spleen and liver where they are destroyed. The macrophage mediated killing of neutrophils termed as efferocytosis elicits immune modulatory properties anti-inflammatory signaling, prevents neutrophil lysis, and dampens immune responses.

Efferocytosis of apoptotic neutrophils is a fundamental, innate process required for tissue homeostasis and immunity, and its dysregulation can lead to unwanted excessive inflammation, autoimmunity, and exacerbation of infection. Impaired neutrophil efferocytosis has been observed in many human inflammatory lung diseases such as cystic fibrosis, chronic obstructive pulmonary disease, asthma, idiopathic pulmonary fibrosis, glomerulonephritis, atherosclerosis, lung cancer, autoimmune diseases, such as rheumatoid arthritis and systemic lupus erythematous ${ }^{(14,15)}$. Resolution of inflammation is a battle between neutrophils and macrophages, whereby macrophages clear all the neutrophils from the site.

Neutrophils rapidly migrate in high numbers to sites of inflammation (e.g., infection, tissue damage, and cancer) and are subsequently able to migrate to draining lymph nodes $(\mathrm{LNs})^{(16)}$. Once in the tissues, neutrophils perform phagocytic killing of pathogen, undergo apoptosis and accumulate as uncleared apoptotic cells ${ }^{(17)}$. The time at which viable neutrophil numbers are maximum in the tissue is often considered the "peak" of a self-limiting inflammatory episode, that typically coincides with the maximum number of uncleared apoptotic neutrophils in the tissue. This is accompanied by proresolving process and repair of injured epithelium followed by macrophage mediated efferocytosis and clearance of neutrophils and tissue debris during the resolution stage .

All cancers contain cells with malignant morphological features termed as tumor cells, admixed with stromal cells and endothelial cells that constitute the tumor micro environment. Stromal cells, blood vessels and infiltrating inflammatory cells are major components of the tumor microenvironment Sustained reciprocal communications with neoplastic cancer cells and immune cells create the inflammatory tumor microenvironment. The cellular infiltrate composed of mast cells, dendritic cells, $\mathrm{T}$ lymphocytes , natural killer cells (NK), T regulatory cells (Tregs), myeloid derived suppressor cells (MDSC), tumorassociated macrophages (TAM) and tumorassociated neutrophils (TANs) are the key participants of the tumor micro environment where they can promote or inhibit cancer formation and development $^{(18)}$. Cytokines and chemokines 
released from neutrophils recruit other immune cells to the site of injury. .Neutrophils in tumor microenvironment forming an integral part of the battle between immune system and cancer stem cells play complex pro tumorigenic, angiogenic ${ }^{(19)}$, metastatic and antitumorigenic functions. Dual pro and antiinflammatory role of neutrophils in human cancer has been a subject of great interest. Neutrophils have direct action on premalignant epithelial cells in accelerating tumorigenesis. Neutrophils present in tumorsare recruited from blood bychemoattractants secreted by tumor cells.

\section{Role of neutrophils in cancer related inflammation}

Neutrophils (TANs) in tumor microenvironment have been suggested as key players in malignant transformation, tumor progression, antitumoral immunity, and angiogenesis ${ }^{(20)}$ Neutrophils attracted to sites of inflammation by chemokines exert their action by different mechanisms such as cytoplasmic granules, release of a variety of molecules with pro inflammatory or antiinflammatory effects. These include antimicrobial peptides, reactive oxygen species (ROS) and enzymes. Neutrophil extracellular trap formation $\left(\mathrm{NET}_{\text {osis }}\right)$ and neutrophil apoptosis are two major suicidal programmes in tumor microenvironment that has major implications in pathogenesis of neutrophil mediated chronic inflammatory diseases, which remainas major hurdles in understanding thepathogenesis of cancer related inflammation. Neutrophils that have phagocytosed the pathogens are removed by macrophages by a process called efferocytosis. Neutrophil macrophage interaction in tumor micro environment is mediated via various inter cellular signals. A neutrophilic response is having worse prognosis by inhibiting the immune system by way of suppression of cytotoxic $\mathrm{T}$ cells. Tissue infiltration is accompanied by leucocytosis.

Neutrophil lifespan is altered in cancer, extending from 7 hin normal conditions to $17 \mathrm{~h}$ in cancer ${ }^{(4)}$. The immune infiltrating cells in the tumorstroma is critical in tumor behaviour, and response to therapy. The prognostic value of absence, presence or abundance of tumor associated neutrophils (TANs) vary among tumor types and among individuals with same type of tumors or between different tumors in same individual. Increased number of neutrophils in tumor environment is reflected as high neutrophil lymphocyte ratio (NLR) in peripheral blood $^{(4)}$. The tumor microenvironment deregulates neutrophils, mainly via Granulocyte colony stimulating factor (G-CSF) and its receptor $\mathrm{G}-\mathrm{CSFR}^{(12)}$. G-CSF is released from various cell types in the tumor milieu such as endothelial cells, fibroblasts, monocytes and macrophages in response to Toll like receptor (TLR) ligands and pro inflammatory cytokines. Neutrophils and progenitor cells carry surface receptor for G-CSF. Binding to these receptors activates downstream Janus Kinase (JAK) or signal transducer and activation of transcription 3 (STAT-3 pathway). This pathway is an essential signalling pathway for cancer related inflammation. JAK/ STAT3 pathway activation leads to expression of genes required for granulopoesis such as myc, CEBPB, elevated GCSF and GM-CSF have been documented in various human cancers in which high leukocyte count with neutrophil predominance or leukaemoid blood picture is observed.

Protumoral effects of neutrophils are exertedvia matrix metalloproteinases, proteases and reactive oxygen species (ROS). ROS can came DNA damage, render genetic instability, initiate mutation and drive oncogenic transformation in precancerous cells. Tumor progression by neutrophils is by release of enzymes such as neutrophil elastase, (ELA2) which activate phosphoinositide-3 kinase (PI3K) to promote tumor cell proliferation. Activated neutrophils during degranulation release cell surface-derived vesicles, called ectosomes, that expose phosphatidyl serine on their surface, an "eat me" signal for DCs. Neutrophils play critical role in pathogen clearance in inflammation and wound healing. Both at the site of inflammation as well as in the LNs, neutrophils can engage with lymphocytes and antigen-presenting cells ${ }^{(16)}$.

Neutrophils can also prevent recruitment of other innate immune cells to lymph nodes. The pathogen particles transported by neutrophils to secondary 
lymphoid organs are processed and presented to B and $\mathrm{T}$ lymphocytes to stimulate adaptive immunity. Until recently neutrophils were considered to be terminally differentiated cells destined to die at the site of infection. Recent research has shown that neutrophils carrying antigens are the first cells to arrive the draining lymph nodes from the periphery and upregulate molecules associated with antigen presentation and co-stimulation to recruit and activate other cells Such as dendritic cells (DCs). DCs are key players in the initiation of the antigenspecific immune response, bridging innate and adaptive immunity. During neutrophil degranulation, alarmins are also released .Alarmins are damage associated molecular patterns (DAMPs) contributing to the chemoattraction of inflammatory DCs(iDCs). DAMPs act either by a direct action viadefensins and alarmins-like HMGB1 or indirectly through induction of chemokines involved in the recruitment of iDCs such as and CCL5. In addition, neutrophils release proteases, such as cathepsin $\mathrm{G}$ and elastase, that can process inactive, nonclassicalchemokines, such as prochimerin, into chemoattractive molecules.

Heterogenous role of TANs in tumors, needs to be more clearly understood. Aberrant accumulation of neutrophils has been observed in a wide variety of malignant tumors, the prognostic value of which appears non uniform among patients with same type of cancers. In most cases TAN is often associated with poor prognosis and shows positive correlation with advanced tumor size and grade. Conversely large number of tumor infiltrating neutrophils is found to correlate with enhanced survival in early stage lung cancer and in women but not men with advanced gastric cancer. In uterine epithelial cancer, neutrophils have been shown to slow down early tumor growth and progression by inducing tumor cell detachment from the basement membrane. The multi factorial role played by neutrophils in tumor microenvironment has major implications intumor pathogenesis and progression. Th17 type of CD4+ $\mathrm{T}$ cell adaptive immunity in cancer is pivotal in neutrophil recruitment in tumors. IL-17A exerts this effect by orchestrating the local release of neutrophil-mobilising factors in resident cells. Recent studies promote IL-17A as a candidate cytokine for linking the activation of $\mathrm{T}$ lymphocytes to the sustained accumulation and activity of neutrophils in the lungs ${ }^{(6)}$. The association of tumor infiltrating neutrophils with Th17type of adaptive immunity in mediating cancer related inflammation is a subject of enormous interest in cancer research. A number of recent meta-analyses identified TAN as well as high neutrophil-lymphocyte ratio in the blood as one of the most powerful immunologic prognostic parameters in human oncology .Further research to understand the mechanism and functionality of TAN in cancer are encouraged ${ }^{(19)}$.

\section{Limitations}

This is not a prospective study. Correlation with tumor stage was not assessed. Follow up study to find out the correlation with treatment and prognosis was not done. A major hindrance to the follow up study is that most of the cytotoxic drugs used for chemotherapy induce neutropenia. Hence follow up study would be possible only in patients treated with specific receptor blockers such as tyrosine kinase inhibitors orsimilar molecules.

\section{Conclusion}

This study shows that neutrophils are the predominant leukocytes in peripheral blood of lung cancer patients, supporting the concept of ongoing injury and repair processes in the pathogenesis of inflammation in lung cancer. NLR is a simple and inexpensive bed side biomarker of systemic inflammation in lung cancer patients.

\section{Conflict of interest- nil}

\section{References}

1. Forget P, Khalifa C, Defour J-P, Latinne D, Van Pel M-C, De Kock M. What is the normal value of the neutrophil-to-lymphocyte ratio? BMC Res Notes [Internet]. 2017 Jan;10:12

2. Gosens R, Giangreco A, Sahai E, Chambers RC. Mechanistic overlap between chronic lung 
injury and cancer: ERS Lung Science Conference 2017 report. Eur Respir Rev. 2017 Jun 30;26(144): 170060.

3. Sankowski R, Mader S, Vald Ã@s-Ferrer SI. Systemic Inflammation and the Brain: Novel Roles of Genetic, Molecular, and Environmental Cues as Drivers of Neuro degeneration. Front Cell Neurosci. 2015 Feb;9:28.

4. Ocana A, Nieto-Jiménez C, Pandiella A, Templeton AJ. Neutrophils in cancer: prognostic role and therapeutic strategies. Mol Cancer. 2017 Aug;16:137.

5. Gernez Y, Tirouvanziam R, Chanez P. Neutrophils in chronic inflammatory airway diseases: can we target them and how? Eur Respir J. 2010 Mar;35(3):467-9.

6. Linden A. Neutrophils, interleukin-17A and lung disease. Eur Respir J. 2005 Jan;25(1):159_ 72.

7. He X, Zhou T, Yang Y, Hong S, Zhan J, Hu Z, et al. Advanced Lung Cancer Inflammation Index, a New Prognostic Score, Predicts Outcome in Patients With Small-Cell Lung Cancer. Clin Lung Cancer. 2015 Nov;16(6):e165-71.

8. Stotz M, Gerger A, Eisner F, Szkandera J, Loibner H, L Ress A, et al. Increased neutrophil-lymphocyte ratio is a poor prognostic factor in patients with primary operable and inoperable pancreatic cancer. $\mathrm{Br} \mathrm{J}$ Cancer. 2013 Jul;109(2):416-21.

9. Aref $\mathrm{H}$, Refaat $\mathrm{S}$. CRP evaluation in non-small cell lung cancer. Egypt $\mathrm{J}$ Chest Dis Tuberc. 2014 Jul;63(3):717-22.

10.Bagley SJ, Kothari S, Aggarwal C, Bauml JM, Alley EW, Evans TL, et al. Pretreatment neutrophil-to-lymphocyte ratio as a marker of outcomes in nivolumab-treated patients with advanced non-small-cell lung cancer. Lung Cancer Amst Neth. 2017;106:1-7.

11.Diem S, Schmid S, Krapf M, Flatz L, Born D, Jochum W, et al. Neutrophil-to-Lymphocyte ratio (NLR) and Platelet-to-Lymphocyte ratio (PLR) as prognostic markers in patients with non-small cell lung cancer (NSCLC) treated with nivolumab. Lung Cancer Amst Neth. 2017;111:176-81.

12. Serhan CN, Brain SD, Buckley CD, Gilroy DW, Haslett C, O'Neill LAJ, et al. Resolution of inflammation: state of the art, definitions and terms. FASEB J. 2007 Feb;21(2):325-32.

13. Ibrahim SA, Kulshrestha A, Katara GK, Beaman KD. Delayed neutrophil apoptosis is regulated by cancer associated a2 isoform vacuolar ATPase. J Immunol. 2017 May 1;198(1 Supplement):76.15-76.15.

14.Greenlee-Wacker MC. Clearance of apoptotic neutrophils and resolution of inflammation. Immunol Rev. 2016 Sep;273(1):357-70.

15. Dehle FC, Mukaro VR, Jurisevic C, Moffat D, Ahern J, Hodge G, et al. Defective Lung Macrophage Function in Lung Cancer \pm Chronic Obstructive Pulmonary Disease (COPD/Emphysema)-Mediated by Cancer Cell Production of PGE2? PLOS ONE. 2013 Apr 26;8(4):e61573.

16.Leliefeld PHC, Koenderman L, Pillay J. How Neutrophils Shape Adaptive Immune Responses. Front Immunol. 2015 Sep;6:471.

17.Elliott MR, Koster KM, Murphy PS. Efferocytosis signaling in the regulation of macrophage inflammatory responses. J Immunol Baltim Md 1950. 2017 Feb;198(4):1387-94.

18. Shen M, Hu P, Donskov F, Wang G, Liu Q, Du J. Tumor-Associated Neutrophils as a New Prognostic Factor in Cancer: A Systematic Review and Meta-Analysis. Coleman WB, editor. PLoS ONE. 2014 Jun 6;9(6):e98259.

19.Liang W, Ferrara N. The Complex Role of Neutrophils in Tumor Angiogenesis and Metastasis. Cancer Immunol Res. 2016 Feb;4(2):83-91.

20.Kim J, Bae J-S. Tumor-Associated Macrophages and Neutrophils in Tumor Microenvironment. Mediators Inflamm. 2016. 